\title{
A Hot-Spot Mutation in CDC42 (p.Tyr64Cys) and Novel Phenotypes in the Third Patient with Takenouchi-Kosaki Syndrome
}

\author{
Midori Motokawa ${ }^{1}$, Satoshi Watanabe ${ }^{1}$, Akiko Nakatomi ${ }^{1}$, Tatsuro Kondoh ${ }^{2}$, Tadashi \\ Matsumoto $^{2}$, Kanako Morifuji ${ }^{3}$, Hirotake Sawada ${ }^{4}$, Toyoki Nishimura ${ }^{4}$, Hiroyuki \\ Nunoi $^{4}$, Koh-ichiro Yoshiura ${ }^{5}$, Hiroyuki Moriuchi ${ }^{1}$, and Sumito Dateki ${ }^{*}$ \\ ${ }^{1}$ Department of Pediatrics, Nagasaki University Graduate School of Biomedical Sciences, \\ Nagasaki, Japan \\ ${ }^{2}$ Misakaenosono Mutsumi Developmental, Medical, and Welfare Center, Isahaya, Japan \\ ${ }^{3}$ Department of Nursing, Nagasaki University Graduate School of Biomedical Sciences, \\ Nagasaki, Japan \\ ${ }^{4}$ Division of Pediatrics, Department of Reproductive and Developmental Medicine, Faculty \\ of Medicine, University of Miyazaki, Miyazaki, Japan \\ ${ }^{5}$ Department of Human Genetics, Nagasaki University Graduate School of Biomedical \\ Sciences, Nagasaki, Japan
}

Running title: CDC42 mutation and TKS

Number of text pages: 11

Number of abstract words: 147 words

Number of text words: 1106 words

Number of references: 10

Figures: 2

*Correspondence to:

Sumito Dateki, M.D.

Department of Pediatrics, Nagasaki University Graduate School of Biomedical Sciences

Address: 1-7-1 Sakamoto, Nagasaki, 852-8501 Japan

E-mail: sdateki1@nagasaki-u.ac.jp

Phone: +81-95-819-7298

FAX: $+81-95-819-7301$ 


\section{ABSTRACT}

Takenouchi-Kosaki syndrome (TKS) is a congenital malformation syndrome characterized by severe developmental delay, macrothrombocytopenia, camptodactyly, sensorineural hearing loss, and dysmorphic facial features. Recently, a heterozygous de novo mutation (p.Tyr64Cys) in the CDC42 gene, which encodes a key small GTP binding protein of the Rho-subfamily, was identified in two unrelated patients with TKS. We herein report a third patient with TKS who had the same heterozygous CDC42 mutation. The phenotype of the patient was very similar to those of the two previously reported patients with TKS; however, she also demonstrated novel clinical manifestations, such as congenital hypothyroidism and immunological disturbance. Thus, despite the heterozygous mutation of CDC42 (p.Tyr64Cys) likely being a hot-spot mutation for TKS, its phenotype may be variable. Further studies and the accumulation of patients with CDC42 mutations are needed to clarify the phenotype in patients with TKS and the pathophysiological roles of the CDC42 mutation.

Keywords: Takenouchi-Kosaki syndrome, CDC42, immunodeficiency, congenital hypothyroidism 


\section{INTRODUCTION}

Takenouchi-Kosaki syndrome (TKS) is a rare congenital malformation syndrome characterized by severe developmental delay, macrothrombocytopenia, camptodactyly, sensorineural deafness, and dysmorphic facial features (OMIM 616737). ${ }^{1,2}$ Although only two unrelated patients with TKS have been reported thus far, both of them were heterozygous for the same de novo CDC42 mutation (p.Tyr64Cys). ${ }^{1,2}$

CDC42 encode a key small GTP binding protein of the Rho-subfamily, which regulates signaling pathways that control various cellular functions, including cell morphology, migration, endocytosis, and cell cycle progression; ${ }^{3}$ however, the mechanisms underlying the CDC42 mutation leading to the variable phenotype of TKS remain to be determined.

We herein report a third patient with TKS who had exactly the same missense mutation in CDC42 reported previously and broader clinical manifestations than the first two patients. The present observation strongly suggests that the p.Tyr64Cys variant in CDC42 is a hot-spot mutation for TKS that may cause broader phenotype than previously thought. 


\section{CASE REPORTS}

A Japanese female patient was born to non-consanguineous parents at 35 weeks of gestation.

During the gestation period, hydrops and pleural effusion had been detected by fetal echography, but improved spontaneously toward birth. At birth, her length was $46.3 \mathrm{~cm}$ $(+0.35$ standard deviations [SD]), weight $2.34 \mathrm{~kg}(+0.38 \mathrm{SD})$, and OFC $31.5 \mathrm{~cm}(-0.10 \mathrm{SD})$.

She had distinctive facial features consisting of midface hypoplasia, low-set ears, synophrys, mild ptosis, slender eyes, exotropia, and thin upper lip (Figure 1a). She also had camptodactyly of bilateral fingers (Figure 1b), hallux valgus (Figure 1c), sensorineural deafness and congenital hypothyroidism detected by a neonatal mass screening. Initially, her serum thyroid-stimulating hormone level was $85.61 \mu \mathrm{IU} / \mathrm{mL}$ [normal range: $0.35-4.94$ ] and free triiodothyronine level $0.76 \mathrm{ng} / \mathrm{dL}$ [0.70-1.48]. The thyroid ultrasonography showed adequately located thyroid gland of normal size. She started treatment with levothyroxine sodium $(10 \mu \mathrm{g} / \mathrm{kg} /$ day) that normalized her thyroid function promptly. She had severe motor developmental delay. She held up her head at 4 months, rolled over at 6 months, and walked alone at 3 years of age. The brain magnetic resonance imaging examination revealed corpus callosum hypoplasia, dysmyelination and enlarged cerebral ventricles (Figure 2a/2b). A skeletal survey showed maxillary hypoplasia (Figure 2c) and flexion contracture of the proximal interphalangeal joints except for thumbs (Figure 2d).

She often developed bacterial lower respiratory tract infections that sometimes needed 
hospitalization. An immunological examination revealed low serum immunoglobulin levels (IgG $527 \mathrm{mg} / \mathrm{dL}$ [870-1700], IgM $107.0 \mathrm{mg} / \mathrm{dL}$ [35-220], and IgA $64 \mathrm{mg} / \mathrm{dL}$ [110-410]) at 4 years of age. The hypogammagloburinemia has been persistent. Repeated vaccination against measles, rubella, and varicella-zoster viruses failed to induce antibodies to the respective viruses. A lymphocyte subset analysis revealed CD4 $4^{+} \mathrm{T}$-lymphopenia $\left(140 / \mathrm{mm}^{3}, 25.3 \%\right)$ and B-lymphopenia $\left(37 / \mathrm{mm}^{3}, 6.6 \%\right)$.

She was found to have thrombocytopenia $(95,000 / \mu \mathrm{L})$ at 2 years and 6 months of age with a normal hemoglobin concentration and a normal white blood cell count by a routine blood examination. Platelet counts have been around 100,000/ $\mu \mathrm{L}$ thus far. Bone marrow aspiration demonstrated normal nucleated cell counts and the absence of abnormal cells. A peripheral blood smear revealed enlarged platelets (Figure 1d). The results of a platelet aggregation test and bleeding time were within the normal ranges.

On the last examination at 12 years of age, she was $141.8 \mathrm{~cm}(-1.65 \mathrm{SD})$ tall and weighed $32.5 \mathrm{~kg}(-1.31 \mathrm{SD})$. She had severe intellectual disability (total IQ 42), but could communicate with simple words and did not have any autistic and behavioral problems. She needs daily special care from her parents and has attended to a special-needs school.

\section{MOLECULAR STUDIES}

This study was approved by the Institutional Review Board at Nagasaki University Graduate 
School of Biomedical Sciences. After obtaining written informed consent, leukocyte genomic DNA was obtained from the patient and her parents. Firstly, we performed trio whole-exome sequencing for the family; however, no candidate mutations were detected after the mutation annotation process. Subsequently, we performed PCR direct sequencing for the coding exons and their flanking splice sites in CDC42, because her phenotype was very similar to those of previously reported patients with TKS. The primer sequences are available on request. The direct capillary sequencing identified a de novo heterozygous mutation in exon 5 of CDC42 (c.191A>G, p.Tyr64Cys) (NM_001039802.1) in the patient.

\section{DISCUSSION}

We herein report the third patient with TKS. The CDC42 missense mutation was the same as those identified in the two previously reported unrelated patients. Because a part of CDC42 including the mutation site lies within the segmentally duplicated region (http://genome.ucsc.edu/), whole-exome sequencing screening may fail to detect mutations in CDC42 after the mutation annotation process which automatically excludes the mutations in the segmental duplications.

The amino acid tyrosine at position 64 in Cdc42 represents the phosphorylation target for the epidermal growth factor-dependent activation of $\mathrm{Cdc} 42$, which regulates downstream signal transduction. ${ }^{4}$ Although the pathological mechanism of the mutant protein 
remains unknown, the p.Tyr64Cys in CDC42 is likely a hot-spot mutation leading to the various manifestations of TKS.

The clinical findings in the previously reported and present patients with TKS highlight several interesting findings. First, hydrops and pleural effusion were observed in her fetal stage. Since lymphedema is a feature shared with the two previously reported patients, the development of the fetal hydrops in the present patient may be associated with lymphedema in fetus. Second, although macrothrombocytopenia is a pathognomonic manifestations for TKS, the absence of a bleeding tendency, normal platelet aggregation ability, and normal bleeding time in the patient indicate that the phenotype is clinically insignificant. Third, our patient presented with congenital hypothyroidism as a novel phenotype for TKS. In this regard, Cdc42 could be required for epithelial polarity and organization in the endoderm as well as apical constriction in the thyroid bud, and outgrowth of the thyroid buds is severely curtailed in Cdc42-deficient mouse embryo. ${ }^{5}$ These findings suggest that thyroid dysfunction may be associated with CDC42 mutation. Futhermore, the primary vaccine failures along with hypogammaglobulinemia, B-lymphopenia, and $\mathrm{CD} 4^{+} \mathrm{T}-$-lyphopenia in the present patient may also be associated with the CDC42 mutation for the following reasons: CDC42 directly binds to a GTPase-binding domain of Wiskott-Aldrich syndrome protein, the defect of which leads to immunodeficiency associated with abnormal immunoglobulin levels and T-cell dysfunction. ${ }^{6}$ In addition, Cdc42 plays an 
important role in B-cell motility and filopodia formation as well as the development and maturation of B-cell lineage. ${ }^{7.8 .9}$ Deletion of $\mathrm{Cdc} 42$ in mice leads to reduction of mature B-cell populations and production of antigen-specific IgM, IgG1 and IgG3. ${ }^{10}$

In conclusion, we present a third patient with TKS. The heterozygous mutation of CDC42 (p.Tyr64Cys) is likely a hot-spot mutation for TKS. Further studies and the accumulation of patients with CDC42 mutations are needed to clarify the phenotype in patients with TKS and the pathophysiologic roles of the CDC42 mutation.

\section{CONFLICTS OF INTEREST}

The authors declare no conflicts of interest.

\section{ACKNOWLEDGEMENTS}

We thank the patient and the family who participated in this study. This work was supported by a grant for the Initiative on Rare and Undiagnosed Diseases in Pediatrics (no. 15gk0110012h0101) from the Japan Agency for Medical Research and Development (AMED), Tokyo, Japan. 


\section{REFERENCES}

1. Takenouchi, T., Kosaki, R., Niizuma, T., Hata, K. Kosaki, K. Macrothrombocytopenia and developmental delay with a de novo CDC42 mutation: Yet another locus for thrombocytopenia and developmental delay. Am. J. Med. Genet. A. 167A, 2822-2825 (2015).

2. Takenouchi, T., Okamoto, N., Ida, S., Uehara, T. Kosaki, K. Further evidence of a mutation in $\mathrm{CDC} 42$ as a cause of a recognizable syndromic form of thrombocytopenia. Am. J. Med. Genet. A. 170A, 852-855 (2016).

3. Etienne-Manneville, S. Hall, A. Rho GTPases in cell biology. Nature. 420, 629-635 (2002).

4. Tu, S., Wu, W.J., Wang, J., Cerione, R.A. Epidermal growth factor-dependent regulation of Cdc42 is mediated by the Src tyrosine kinase. J. Biol. Chem. 278, 49293-49300 (2003).

5. Loebel, D.A., Plageman, T.F., Jr., Tang, T.L., Jones, V.J., Muccioli, M. Tam, P.P. Thyroid bud morphogenesis requires CDC42- and SHROOM3-dependent apical constriction. Biol Open. 5, 130-139 (2016).

6. Abdul-Manan, N., Aghazadeh, B., Liu, G.A., Majumdar, A., Ouerfelli, O., Siminovitch K,A., et al. Structure of Cdc42 in complex with the GTPase-binding domain of the 'Wiskott-Aldrich syndrome' protein. Nature. 399, 379-383 (1999). 
7. Yoshida, H., Tomiyama, Y., Ishikawa, J., et al. Integrin-associated protein/CD47 regulates motile activity in human B-cell lines through CDC42. Blood. 96, 234-241 (2000).

8. Westerberg, L., Greicius, G., Snapper, S.B., Aspenström, P., Severinson, E. Cdc42, Rac1, and the Wiskott-Aldrich syndrome protein are involved in the cytoskeletal regulation of B lymphocytes. Blood. 98, 1086-1094 (2001).

9. Grill, B., Schrader, J.W. Activation of Rac-1, Rac-2, and Cdc42 by hemopoietic growth factors or cross-linking of the B-lymphocyte receptor for antigen. Blood. 100, 3183-3192 (2002).

10. Guo, F., Velu, C.S., et al. Rho GTPase Cdc42 is essential for B-lymphocyte development and activation. Blood. 114, 2909-2916 (2009) 


\section{TITLES AND LEGENDS TO FIGURES}

\section{Figure 1. Clinical phenotype of the patient}

(a) Facial feature observed in her infancy. (b, c) Camptodactyly of hands (b) and hallux valgus (c) at the age of 11 years. (d) A peripheral blood smear showing a giant platelet (arrow).

\section{Figure 2. Magnetic resonance imaging and X-ray examinations}

T1-weighted horizontal (a) and T2-weighted sagittal (b) views of the brain show corpus callosum hypoplasia and enlarged cerebral ventricles with cerebral aqueduct stenosis (white arrow). (c) Maxillary hypoplasia and (d) flexion contracture of the proximal interphalangeal joints except for the thumb. 
Figure.1

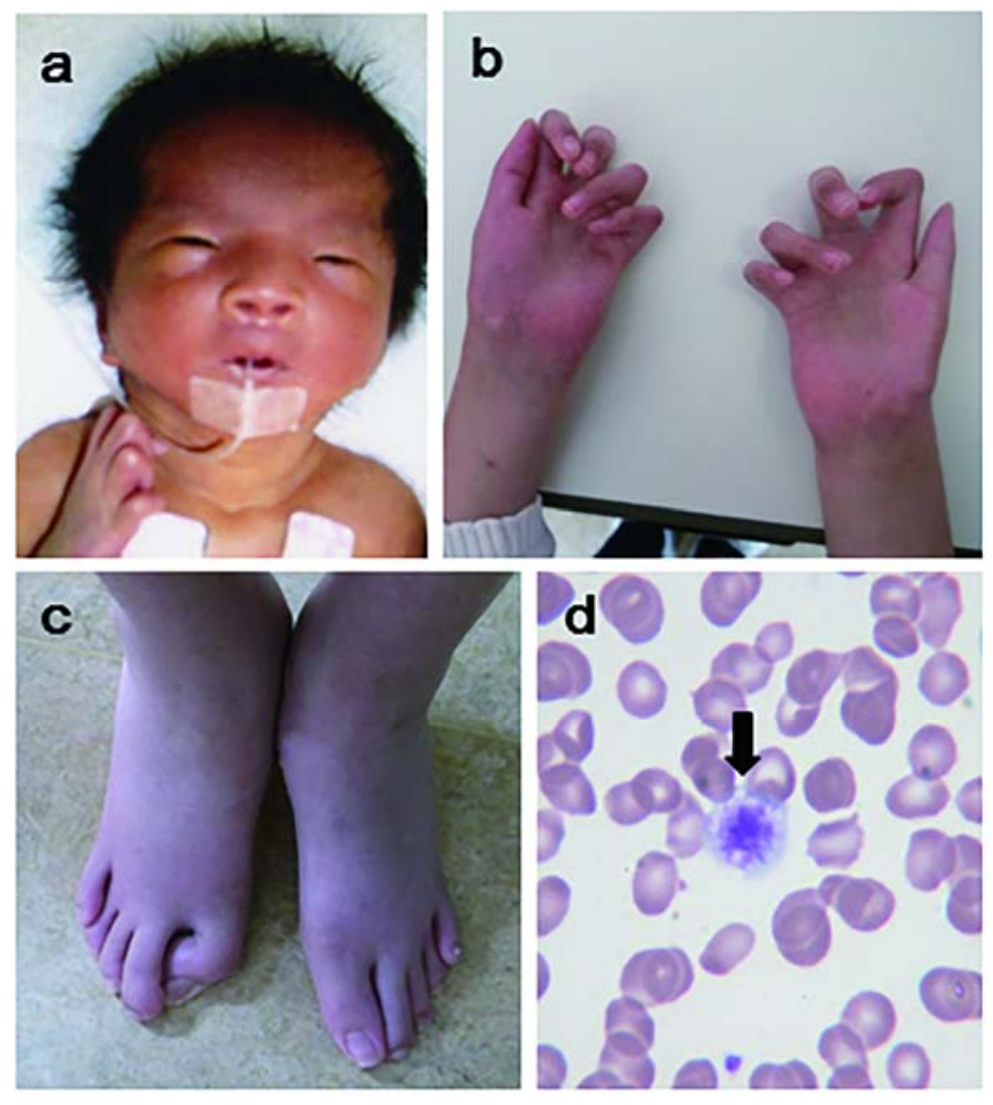


Figure.2

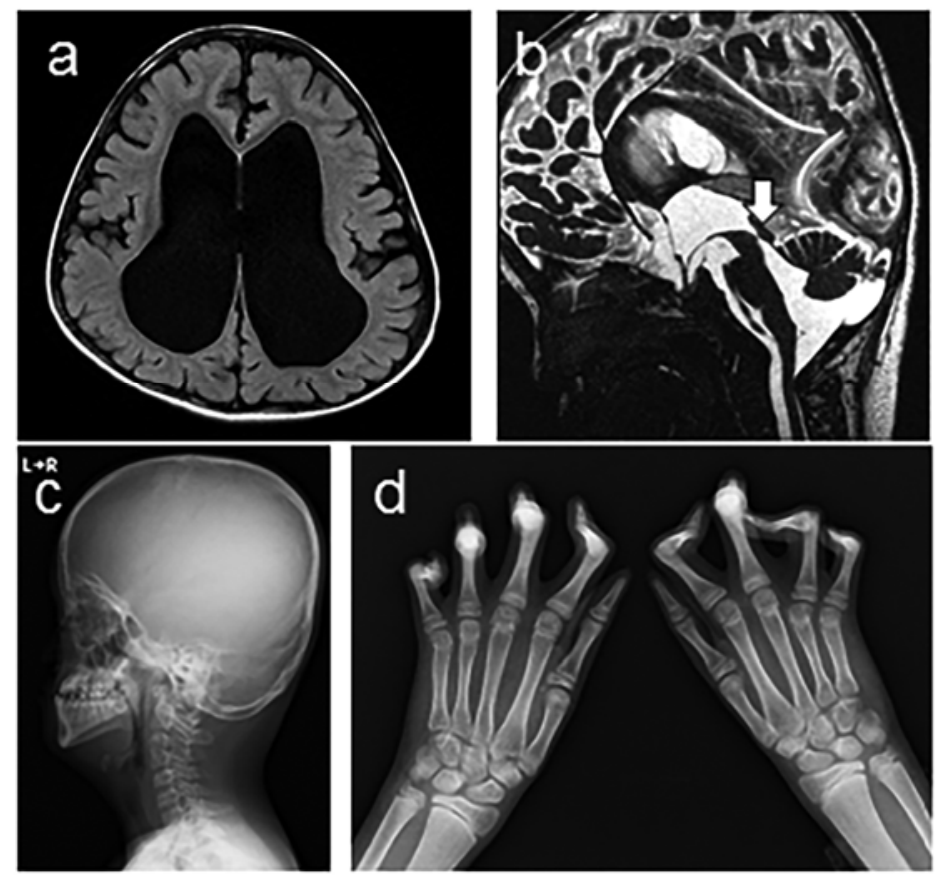

